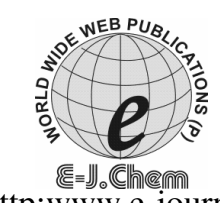

http:www.e-journals.net

ISSN: 0973-4945; CODEN ECJHAO

E-Journal of Chemistry

2011, 8(1), 180-184

\title{
Synthesis, Characterization and Use of Schiff Bases as Fluorimetric Analytical Reagents (Part II)
}

\author{
MOHAMED N. IBRAHIM* and SALAHEDDIN A. I. SHARIF \\ *Chemistry Department, Faculty of Science, Garyounis University, Benghazi, Libya \\ Biology Department, Faculty of Education (Qemeenes Branch) \\ Garyounis University, Benghazi, Benghazi, Libya \\ mnibrahim46@yahoo.com
}

Received 11 November 2009; Accepted 29 December 2009

\begin{abstract}
Many Schiff bases were prepared by condensation reaction of certain aromatic amines with aromatic aldehydes derivatives and then the fluorescence properties of these Schiff bases were examined in acidic and basic media. It is shown that these compounds can be used for fluorimetric monitoring of small $\mathrm{pH}$ changes.
\end{abstract}

Keywords: Schiff bases, Fluorescent indicators, Anthranilic acid, o-phenylenediamine.

\section{Introduction}

Schiff bases derived from aromatic amines and aromatic aldehydes have a wide variety of applications in many fields, e.g., biological, inorganic and analytical chemistry ${ }^{1-5}$. Application of many new analytical devices requires the presence of organic reagents as essential compounds of the measuring system. They are used, e.g., in optical and electrochemical sensors, as well as in various chromatographic methods, to enable detection of enhance selectivity and sensitivity ${ }^{6-8}$.

Among the organic reagents actually used, Schiff bases possess excellent characteristics, structural similarities with natural biological substances, relatively simple preparation procedures and the synthetic flexibility that enables design of suitable structural properities ${ }^{9,10}$.

Schiff bases are widely applicable in analytical determination, using reactions of condensation of primary amines and carbonyl compounds in which the azomethine bond is formed (determination of compounds with an amino or carbonyl group); using complex formation reactions (determination of amines, carbonyl compounds and metal ions); or utilizing the variation in their spectroscopic characteristics following changes in $\mathrm{pH}$ and solvent ( $\mathrm{pH}$ of solvent polarity indicators) ${ }^{11-13}$.

Unfortunately, most Schiff bases are chemically unstable and show a tendency to be involved in various equilibria, like tautomeric interconversions, hydrolysis, or formation of ionized species ${ }^{14,15}$. Therefore, successful application of Schiff bases requires a careful study of their characteristics. 
The Schiff bases prepared are condesation products of aromatic aldehyde derivatives with aromatic mono- and diamines derivatives and presented in (Scheme 1). In this work the spectroscopic characteristics and possibilities of analytical applications of these Schiff bases are presented.

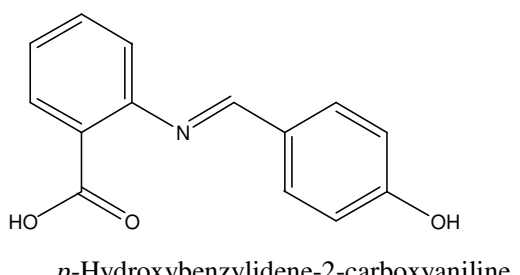<smiles>O=C(O)c1ccccc1/N=C/c1ccc([N+](=O)[O-])cc1</smiles>

p-Nitrobenzylidene-2-carboxyaniline<smiles>CN(C)c1ccc(/C=N/c2ccccc2C(=O)O)cc1</smiles>

$P$-(N,N-Dimethyl)aminobenzylidene-2-carboxyaniline

II

III
I<smiles>Nc1ccccc1/N=C/c1ccc(O)cc1</smiles>

$N$-(4-Hydroxybenzylidene)-benzene-1,2-diamine

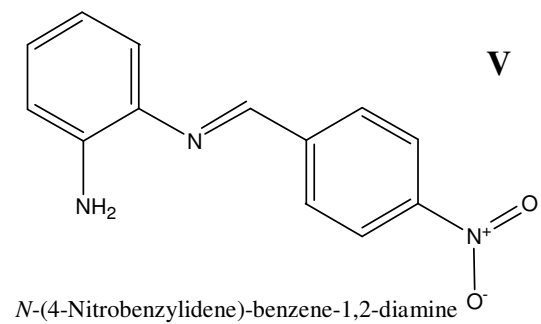

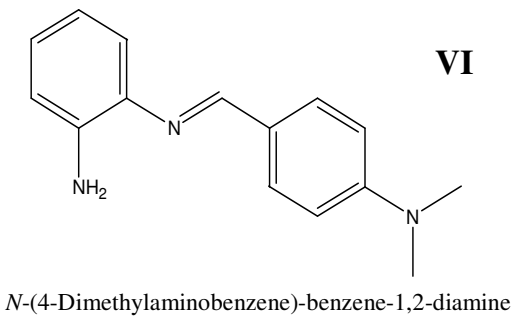

\section{Experimental}

\section{Scheme 1}

All chemicals and solvents used for synthesis were of reagent grade. All melting points were taken on a melting point apparatus and are uncorrected. IR spectra were recorded on a Shimadzu 5000 instrument. ${ }^{1} \mathrm{H}$ NMR were run on a Jeol $500 \mathrm{MHz}$ instrument using TMS as internal standard and DMSO as solvent. The spectral analyses were carried out at the NMR laboratory, Alexandaria University, Alexandaria; the elemental analyses at Microanalytical center Cairo University, Cairo, Egypt

The Schiff bases (I-VI) were prepared according to the reported methods ${ }^{16,17}$. The procedure is as follows: A solution of the amine derivative $(0.01 \mathrm{~mol})$ in absolute ethanol $(10 \mathrm{~mL})$ was slowly added to a solution of the aldehyde derivative $(0.01 \mathrm{~mol})$ in absolute ethanol $(10$ $\mathrm{mL}$ ). After stirring the reaction mixture for $2 \mathrm{~h}$ (compounds I, II and III), and for $5 \mathrm{~h}$ at $60-70{ }^{\circ} \mathrm{C}$ and cooling (compounds IV, $\mathbf{V}$ and $\mathbf{V I}$ ), a precipitate was formed which collected by filtration then washed several times with cold ethanol and recrystallised from ethanol.

$p$-Hydroxybenzylidene-2-carboxyaniline I was prepared from anthranilic acid and p-hydroxybenzaldehyde; pale orange; m.p. 229.5-230 ${ }^{\circ} \mathrm{C} ; 1.6 \mathrm{~g}(70 \%)$ yield. IR $\left(\mathrm{KBr}, \mathrm{cm}^{-1}\right)$ $v=3500(\mathrm{OH}), 2900(\mathrm{C}-\mathrm{H}), 1715(\mathrm{C}=\mathrm{O}), 1615(\mathrm{C}=\mathrm{N}), 1490(\mathrm{C}=\mathrm{C}), 1280,1170(\mathrm{C}-\mathrm{O}) ;{ }^{1} \mathrm{H}$ NMR (500 MHz, DMSO); $\delta(\mathrm{ppm}): 6.41-7.66(8 \mathrm{H}, \mathrm{m}, \mathrm{Ar}-\mathrm{H}), 8.3(1 \mathrm{H}, \mathrm{s}, \mathrm{CH}=\mathrm{N}), 9.70(1 \mathrm{H}, \mathrm{s}$, Ar-OH), $11.00(1 \mathrm{H}, \mathrm{s}, \mathrm{COOH}) ; \mathrm{C}_{14} \mathrm{H}_{11} \mathrm{NO}_{3}(241.2)$ : calcd. C 69.65\%, H 4.56\%, N 5.80\%; found C $68.92 \%, \mathrm{H} 4.52 \%$, N $5.77 \%$. 
$p$-Nitrobenzylidene-2-carboxyaniline (II) was prepared from anthranilic acid and p-nitrobenzaldehyde; yellow-white; m.p. $172-174{ }^{\circ} \mathrm{C} ; 1.7 \mathrm{~g}(62 \%)$ yield. IR $\left(\mathrm{KBr}, \mathrm{cm}^{-1}\right)$ $v=3500(\mathrm{OH}), 2900(\mathrm{C}-\mathrm{H}), 1720(\mathrm{C}=\mathrm{O}), 1610(\mathrm{C}=\mathrm{N}), 1480(\mathrm{C}=\mathrm{C}), 1495,1320(\mathrm{~N}=\mathrm{O})$, 1285,1170(C-O); ${ }^{1} \mathrm{HNMR}$ ( $\left.500 \mathrm{MHz}, \mathrm{DMSO}\right) ; \delta$ (ppm): 6.35-8.28 (8H, m, Ar-H), 8.30 $(1 \mathrm{H}, \mathrm{s}, \mathrm{CH}=\mathrm{N}), 10.11(1 \mathrm{H}, \mathrm{s}, \mathrm{COOH}) ; \mathrm{C}_{14} \mathrm{H}_{10} \mathrm{~N}_{2} \mathrm{O}_{4}(270.2)$ : calcd. C $62.17 \%, \mathrm{H} 3.70 \%, \mathrm{~N}$ $10.36 \%$; found $\mathrm{C} 61.88 \%, \mathrm{H} 3.65 \%$, N $10.03 \%$.

$p$-(N,N-Dimethyl)aminobenzylidene-2-carboxyaniline (III) was prepared from anthranilic acid and $p$ - $(N, N$-dimethylamino $)$ benzaldehyde; pale green; m.p. $198{ }^{\circ} \mathrm{C} ; 2.0 \mathrm{~g}$ $(75 \%)$ yield. IR $\left(\mathrm{KBr}, \mathrm{cm}^{-1}\right) \mathbf{v}=3500(\mathrm{OH}), 2910(\mathrm{C}-\mathrm{H}), 1725(\mathrm{C}=\mathrm{O}), 1615(\mathrm{C}=\mathrm{N}), 1355$ $(\mathrm{C}-\mathrm{N}), 1475(\mathrm{C}=\mathrm{C}) 1280,1170(\mathrm{C}-\mathrm{O}) ;{ }^{1} \mathrm{H}$ NMR (500 MHz, DMSO); $\delta(\mathrm{ppm}): 2.89(6 \mathrm{H}, \mathrm{s}, \mathrm{N}-$ $\left.\left(\mathrm{CH}_{3}\right)_{2}\right), 6.41-7.62(8 \mathrm{H}, \mathrm{m}, \mathrm{Ar}-\mathrm{H}), 8.29(1 \mathrm{H}, \mathrm{s}, \mathrm{CH}=\mathrm{N}), 9.57(1 \mathrm{H}, \mathrm{s}, \mathrm{Ar}-\mathrm{OH}) ; \mathrm{C}_{16} \mathrm{H}_{16} \mathrm{~N}_{2} \mathrm{O}_{2}$ (268.2): calcd. C 71.58\%, H 5.96\%, N 10.43\%; found C 70.42\%, H 5.81\%, N 9.33\%.

$\mathrm{N}$-(4-Hydroxybezylidene)-benzene-1,2-diamine (IV) was prepared from $o$-phenylenediamine and $p$-hydroxybenzaldehyde; cotton pale brown; m.p. $148^{\circ} \mathrm{C} ; 1.3 \mathrm{~g}(64 \%)$ yield. IR $\left(\mathrm{KBr}, \mathrm{cm}^{-1}\right) v=3400,3300(\mathrm{~N}-\mathrm{H}), 3000(\mathrm{O}-\mathrm{H}), 3010(\mathrm{C}-\mathrm{H}), 1600(\mathrm{C}=\mathrm{N}), 1445(\mathrm{C}=\mathrm{C}), 1360$ (C-N); ${ }^{1} \mathrm{HNMR}(500 \mathrm{MHz}, \mathrm{DMSO}) ; \delta(\mathrm{ppm}): 5.00\left(2 \mathrm{H}, \mathrm{s},-\mathrm{NH}_{2}\right), 6.41-7.70(8 \mathrm{H}, \mathrm{m}, \mathrm{Ar}-\mathrm{H})$, $8.38(1 \mathrm{H}, \mathrm{s}, \mathrm{CH}=\mathrm{N}), 10.47(1 \mathrm{H}, \mathrm{s}, \mathrm{Ar}-\mathrm{OH}) ; \mathrm{C}_{13} \mathrm{H}_{12} \mathrm{~N}_{2} \mathrm{O}$ ( 212.2 ): calcd. C 73.51\%, H 5.65\%, N $13.19 \%$; found C $73.01 \%$, H $5.42 \%, \mathrm{~N} 12.87 \%$.

$\mathrm{N}$-(4-Nitrobezylidene)-benzene-1,2-diamine (V) was prepared from o-phenylenediamine and p-nitrobenzaldehyde; pale brown; m.p. $317-318^{\circ} \mathrm{C} ; 1.9 \mathrm{~g}(81 \%)$ yield. IR ( $\mathrm{KBr}$, $\left.\mathrm{cm}^{-1}\right) v=3400,3300(\mathrm{~N}-\mathrm{H}), 3010(\mathrm{C}-\mathrm{H}), 1615(\mathrm{C}=\mathrm{N}), 1440(\mathrm{C}=\mathrm{C}), 1365(\mathrm{C}-\mathrm{N}), 1510,1330$ $(\mathrm{N}=\mathrm{O}) ;{ }^{1} \mathrm{H}$ NMR (500 MHz, DMSO); $\delta(\mathrm{ppm}): 5.00\left(2 \mathrm{H}, \mathrm{s},-\mathrm{NH}_{2}\right), 6.40-8.1(8 \mathrm{H}, \mathrm{m}, \mathrm{Ar}-\mathrm{H})$, $8.38(1 \mathrm{H}, \mathrm{s}, \mathrm{CH}=\mathrm{N}) ; \mathrm{C}_{13} \mathrm{H}_{11} \mathrm{~N}_{3} \mathrm{O}_{2}$ (241.2): calcd. $\mathrm{C} 64.67 \%, \mathrm{H} 4.56 \%, \mathrm{~N} 17.41 \%$; found $\mathrm{C}$ $63.28 \%, \mathrm{H} 4.22 \%, \mathrm{~N} 15.96 \%$.

$N$-(4-(N,N-Dimethylaminobezylidene)-benzene-1,2-diamine (VI) was prepared from $o$-phenylenediamine and $p$-(N,N-dimethylbenzaldehyde; yellow; m.p. 141-142 ${ }^{\circ} \mathrm{C} ; 0.4 \mathrm{~g}$ $(16 \%)$ yield. IR $\left(\mathrm{KBr}, \mathrm{cm}^{-1}\right) v=3460,3370(\mathrm{~N}-\mathrm{H}), 2900,2800(\mathrm{C}-\mathrm{H}), 1600(\mathrm{C}=\mathrm{N}), 1440$ $(\mathrm{C}=\mathrm{C}), 1360(\mathrm{C}-\mathrm{N}) ;{ }^{1} \mathrm{HNMR}$ ( $\left.500 \mathrm{MHz}, \mathrm{DMSO}\right) ; \delta(\mathrm{ppm}): 2.86\left(6 \mathrm{H}, \mathrm{s},-\mathrm{N}-\left(\mathrm{CH}_{3}\right)_{2}\right), 4.97$ $\left(2 \mathrm{H}, \mathrm{s},-\mathrm{NH}_{2}\right), 6.40-7.66(8 \mathrm{H}, \mathrm{m}, \mathrm{Ar}-\mathrm{H}), 8.31(1 \mathrm{H}, \mathrm{s}, \mathrm{CH}=\mathrm{N}) ; \mathrm{C}_{15} \mathrm{H}_{17} \mathrm{~N}_{3}$ (239.2): calcd. C75.25\%, H 7.10\%, N 17.55\%; found C 74.90\%, H 6.58\%, N $17.04 \%$.

\section{Results and Discussion}

\section{Fluorescence studies}

Fluorescent indicators ${ }^{18,19}$ have many applications and are generally employed in cases where colorimetric indicators are difficult to observe or lack sensitivity. Such cases are found in dark, turbid or coloured solutions or titrations in which a precipitate is formed. A flash of light from a fluorescent indicator is much easier to see or measure than the appearance of a weak colour. Fluorescent colours under UV light are often easier to observe than a weak change in colour in an ordinary colour indicator.

Determination with fluorescent indicators may be carried out in a dark room or the use of a view box provided with an entry door. UV light (Herolab, $254 \mathrm{~nm}$, NU-4 KL) was used as a source of radiation. For pH measurements, pH-meter (Mettler-Toledo, MP 220) was used. The change of fluorescent intensity or colour of a compound caused by a change in $\mathrm{pH}$ may be the results of equilibrium shifts

$$
\begin{aligned}
\mathrm{AH} \longleftrightarrow \mathrm{H}^{+}+\mathrm{A}^{-} \\
\mathrm{AH}+\mathrm{hv}_{1} \longleftrightarrow \mathrm{AH}^{*} \longleftrightarrow \mathrm{AH}+\mathrm{hv}_{2} \text { fluorescence }
\end{aligned}
$$


The use of fluorescent indicators in titration of coloured solutions is probably the most prominent of the indicator applications. Table 1 below shows the change in colours according to the $\mathrm{pH}$ change:

Table 1. Acid-base fluorescent Schiff bases

\begin{tabular}{cclc}
\hline Schiff base & pH Range & Flourescent Colour Change & Indicator Solution \\
\hline I & $1.5-2.7$ & non-fl. to pale blue & $0.1 \%$ solution \\
& $2.7-6.5$ & bright blue & in $50 \%$ ethanol \\
& $6.5-12.5$ & dark blue & \\
II & $12.5-14$ & pale blue to blue-green & \\
& $0.8-1.4$ & non-fl. & $0.1 \%$ solution \\
& $1.5-2.7$ & pale blue & in $50 \%$ ethanol \\
& $2.7-6.0$ & bright blue & \\
& $6.0-12.6$ & dark blue & \\
III & $12.6-14$ & pale blue to blue-green & \\
& $0.95-1.4$ & pale blue & $0.1 \%$ solution \\
& $1.45-2.0$ & dark blue & in $50 \%$ ethanol \\
& $2.0-6.0$ & bright blue & \\
IV & $6.0-12.5$ & dark blue & \\
& $12.5-14$ & pale blue to blue-green & $0.2 \%$ solution \\
V & $1-9.2$ & pale blue & in $70 \%$ ethanol \\
& $9.2-14$ & bright blue & $0.2 \%$ solution \\
VI & $1-14$ & non-fl. & in $70 \%$ ethanol \\
& $1.0-6.0$ & pale blue & $0.2 \%$ solution \\
& $6.0-14$ & dark blue & in $70 \%$ ethanol \\
\hline
\end{tabular}

*Most of the colours are based on visual observations

Whereas, the effect of $\mathrm{pH}$ on the statring materials (aromatic amines), listed in the Table 2.

Table 2. Acid-base fluorescent aromatic amine ${ }^{19}$

\begin{tabular}{cccc}
\hline Aromatic amine & $\mathrm{pH}$ & Colour Change & Indicator Solution \\
\hline Anthranilic acid & $1.5-3.0$ & non-fl. to light blue & $0.1 \%$ solution \\
& $4.5-6.0$ & $\begin{array}{c}\text { light blue to dark blue } \\
\text { dark blue to non-fl. }\end{array}$ & in 50\% ethanol \\
& $12.5-14$ & green to non-fl. & $\begin{array}{c}0.2 \% \text { solution } \\
\text { in 70\% ethanol }\end{array}$ \\
\hline
\end{tabular}

\section{Conclusion}

Most Schiff bases prepared in this work through condensation of anthranilic acid and $o$-phenylenediamine with the corresponding aldehyde derivatives show fluorescent properties, therefore, they can be used as fluorescent indicators.

Compounds I-III showed characteristic colour change on pH range 1-6 (acidic medium) as well as in basic medium. Compounds IV and VI also showed colour change over a wide $\mathrm{pH}$ range. Compound $\mathbf{V}$ prove to be not suitable and showed non-fluorescence property. The fluorescence of these compounds is $\mathrm{pH}$ dependent and can be used for monitoring $\mathrm{pH}$. This fact opens an attractive possibility of application in optical sensors where $\mathrm{pH}$ sensitivity is required over limited $\mathrm{pH}$ range. 
Future efforts will be carried out on other types of Schiff bases and study of their applications as optical sensors.

\section{References}

1. Cimerman Z, Miljanic S and Galic N, Croatica Chemica Acta, 2000, 73(1), 81-95.

2. $\quad$ Singh P Goel R L and Singh B P, J Indian Chem Soc., 1975, 52, 958-959.

3. Perry B F Beezer A E Miles R J Smith B W Miller J and Nascimento M G, Microbois., 1988, 45, 181.

4. Elmali A Kabak M and Elerman Y, J Mol Struct., 1999, 477, 151.

5. $\quad$ Patel P R Thaker B T and Zele S, Indian J Chem., 1999, 38A, 563-566.

6. Valcarcel M and Laque de Castro M D, Flow-Throgh Biochemical Sensors; Elsevier: Amsterdam, 1994.

7. Spichiger-Keller U E, Chemical Sensors and Biosensors for Medical and Biological Applications, Wiley-VCH, Weinheim, 1998.

8. Lawrence J F and Frei R W, Chemical Derivatization in Liquid Chromatography; Elsevier: Amsterdam, 1976

9. Patai S (Ed.), The Chemistry of the Carbon-Nitrogen Double Bond, J Wiley \& Sons, London, 1970.

10. Jungreis E and Thabet S, Analytical Applications of Schiff bases, Marcell Dekker, New York, 1969.

11. Metzler C M Cahill A and Metzler D E, J Am Chem Soc., 1980, 102, 6075-6082.

12. Dudek G O and Dudek E P, Chem Commun., 1965, 464.

13. Dudek G O and Dudek E P, J Am Chem Soc., 1966, 88, 2407-2412.

14. Cimerman Z and Stefanac Z, Polyhedron, 1985, 4, 1755.

15. Galic N Cimerman Z and Tomisic V, Anal Chim Acta, 1997, 343, 135.

16. Bigelow L A and Eatough H, Org Synth., 1967, 1, 80.

17. Ibrahim M N Hamad K J and Al-Joroshi S H, Asian J Chem., 2006, 18(13), 2404.

18. White C E and Aragauer R J, Fluorescence Analysis, A practical Approach; Marcel Dekker, Inc.: New York, 1970.

19. Bishop E, Indicators; Pergamon Press Ltd., Headington Hill Hall:Oxford, 1972. 


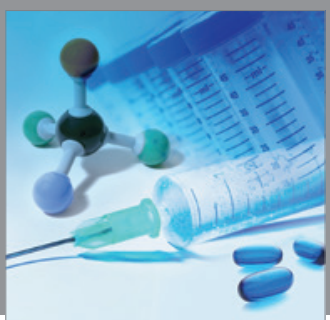

International Journal of

Medicinal Chemistry

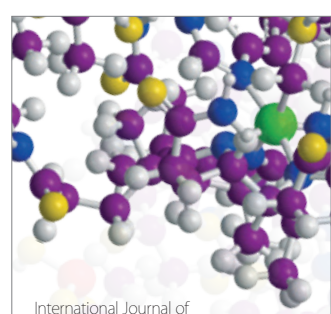

Carbohydrate Chemistry

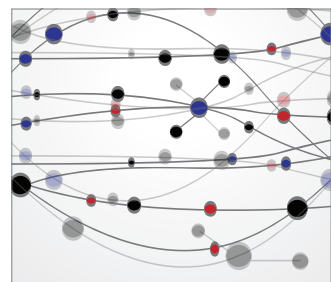

The Scientific World Journal
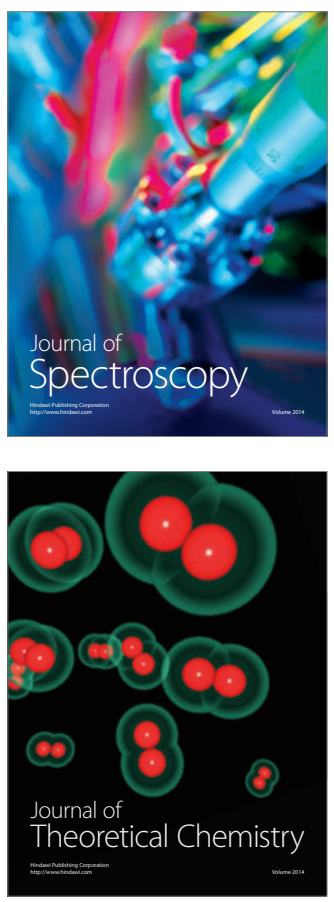
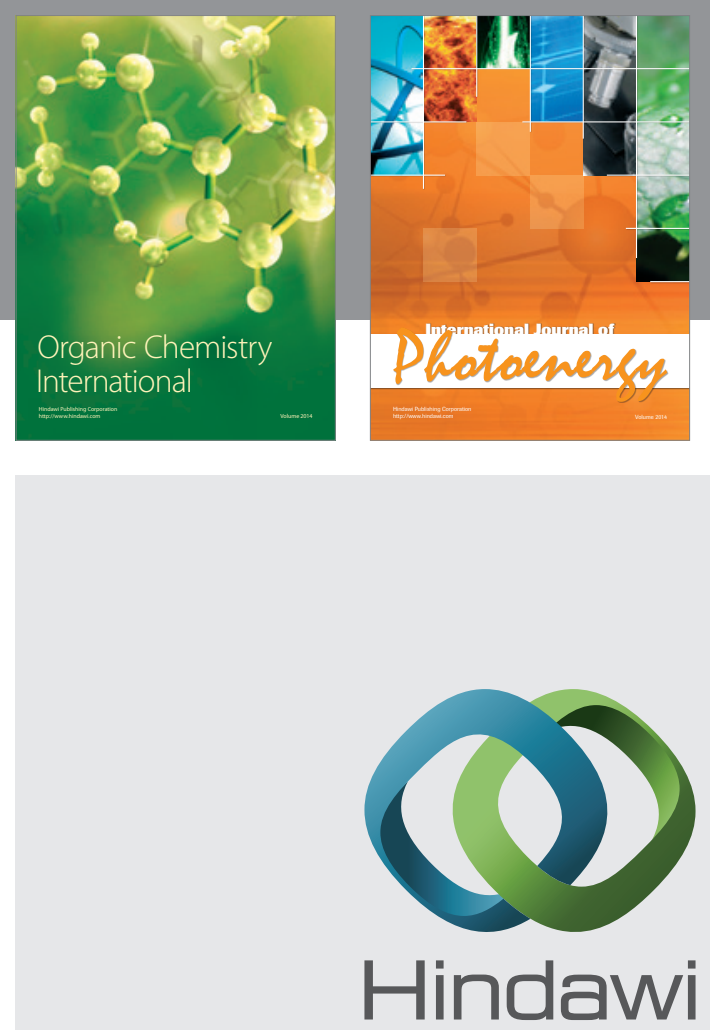

Submit your manuscripts at

http://www.hindawi.com
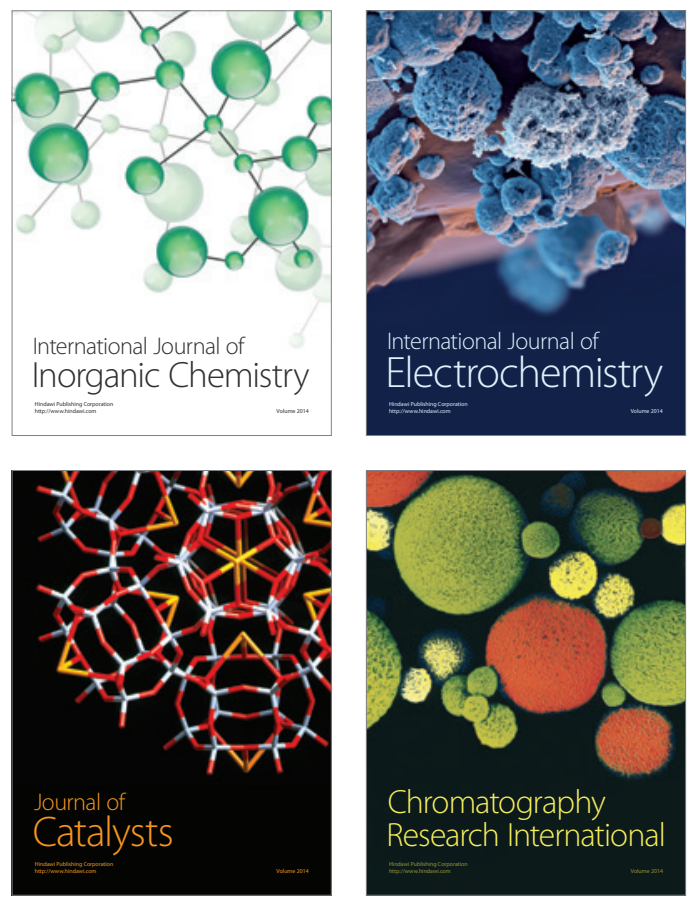
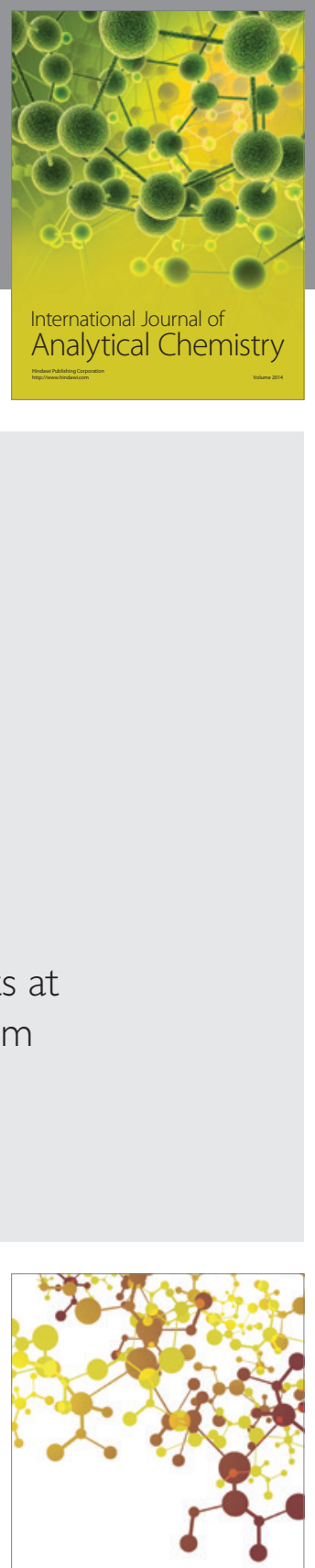

Journal of

Applied Chemistry
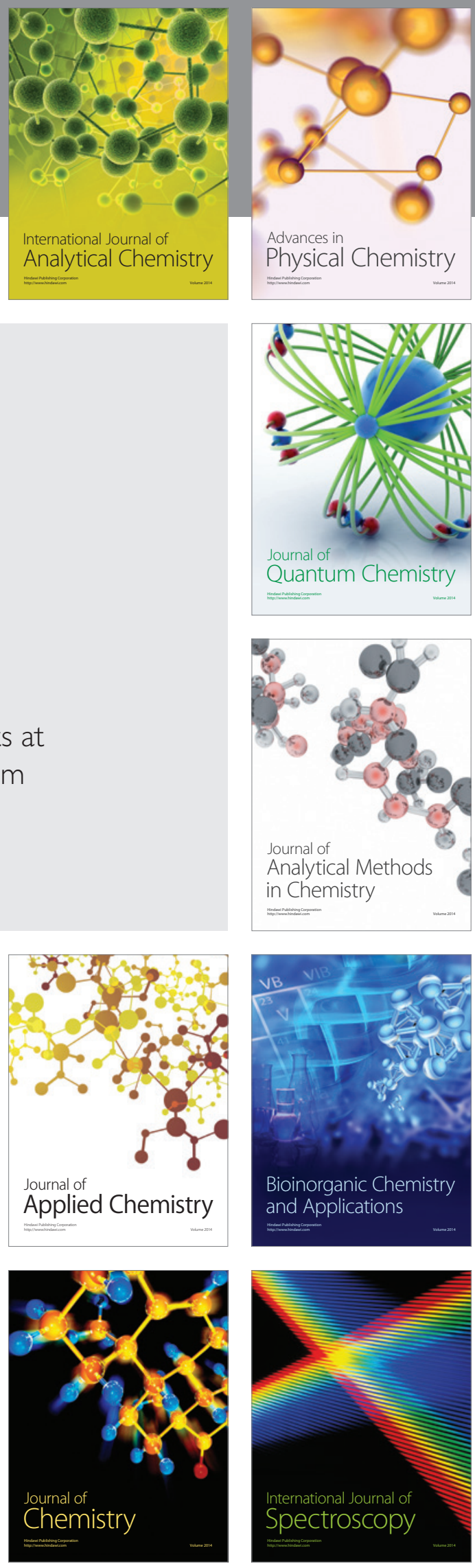